Annals of the

\title{
RHEUMATIC DISEASES
}

Leader

\section{Evaluating new physical treatments}

Physiotherapy as a hospital based specialty first developed out of the practice of nurses massaging their patients, though the practice of manipulation or 'bonesetting' has a much longer history. Earlier techniques were mainly manual, but external means of supplementing manual treatment soon appeared. Increasingly complex arrays of lamps were overtaken in popularity by ways of applying currents of different waveform and wavelength to the body. Latterly that trend has accelerated, and there is now a bewildering array of machinery available to aid the physiotherapist, and commercial pressure to use these newer, ever more expensive, machines is growing fast.

Proof that the considerable financial outlay on machines is justified by their therapeutic advantage is often lacking, however, and the Health Service is no longer a place in which untested clinical opinion can be allowed to drive major purchasing decisions. But acquiring proof of efficacy is not easy, for three possible reasons. Firstly, cynics might argue that there is no evidence for the efficacy of many treatment modalities because they actually do not workthey are simply expensive placebos. Irrespective of the clinical opinions of many therapists, this view cannot be dismissed, mainly because of the two other problems of this area, lack of research expertise and difficulty with trial design and performance. Physiotherapy has come a long way in improving the scientific content of its training, with degree level entry now being the norm. Despite this, many therapists lack both the expertise and the desire to explore their methods in a critical fashion. Those who have the necessary skills and ambition often find formidable obstacles in their way, especially difficulty with funding.

The next problem, that of trial design and performance, is well illustrated by the paper by Heussler et al on page 703 of this journal. They explored the use of one of the newer, more heavily promoted, therapeutic tools, the low power laser. The first problem they encountered was a lack of publications reporting controlled trials, with those that did using varying laser types and varying therapeutic regimens. The more cautious claims of these controlled trials contrasted sharply with the dramatic advantage claimed from the much greater number of uncontrolled studies which had been published. The investigators then confronted the problem of the treatment regimen. In contrast with a drug claiming the same therapeutic effect, which would have included in its regulatory submission at least one dose-ranging study, precise knowledge about optimum use is lacking with most therapeutic machines. Thus an empirical treatment regimen, based on the experience of the participants, had to be incorporated into the trial design. This is the area which will be latched onto by laser protagonists, who will inevitably claim that some small difference in technique would have changed the result dramatically. Such claims should not even be entertained unless they are based on scientifically acceptable controlled observations.

The authors approached the problem of evaluation with a formidable battery of tests, and used the patients as their own controls, the most effective method under the circumstances. The study had two advantages: the absence of any significant physical sensation from the treatment and the rigorous blinding of the therapists. The result illustrated beautifully the need for the study to be rigorously designed and executed. The empirical observation that patients improved was totally justified, but improvement was no greater with active than placebo treatment.

What lessons can be learnt from this study? The Health Service as a whole might ask whether machines should ever be purchased which do not have the same quality of efficacy data available as a new drug-mere proof of electrical safety is not enough. The importance of the placebo effect is not new, but is reinforced. The need for studies such as this to be immaculately designed and executed is clearly shown, as is the total lack of validity of uncontrolled 'studies'.

Physiotherapists must now accept the challenge, stop giving credence to unscientific hype, and concentrate on rigorous evaluation of their work. In doing this, they must have the support of medical and scientific colleagues, encouragement by management, and adequate funding made available through the Health Service and charities sponsoring research. They must be willing to follow research protocols - an impossibility for many of them if my own experience is typical - and they must be willing to discard techniques which fail to prove themselves, however glamorous they may seem. If they fail to do so, and we do not know from the paper whether the authors did so, they will find waiting in the wings nurses who massage their patients.

Aromatherapy and other complementary therapies are increasingly practised by nurses in many specialist areas, and these practitioners are learning fast that they must evaluate their results if their methods are to be accepted. ${ }^{1}$ If even small benefits are shown, the popular image of such 'complementary' therapies is likely to influence purchasers towards buying such techniques as part of their overall 
patient care package. This might well put them into direct competition with physiotherapists, and align them with specialists such as chiropractors, who are already demonstrating their superiority over conventional physiotherapists in some areas of practice. ${ }^{2}$ If physiotherapy is the scientifically based profession it claims to be, it may have to produce proof of its efficacy, not just of its undoubted popularity, very soon, or be supplanted by manipulators and nurses who massage.
South Cleveland Hospital,

Marton Road,

Middlesbrough,

Cleveland TS4 3BW,

United Kingdom

1 Lewith G T, Aldridge D, eds. Clinical research methodology for complementary therapists. London. Hodder and Stoughton, 1993.

therapists. London. Hodder and Stoughton, J, Frank A O. Low back pain of mechanical origin: randomised comparison of chiropractice and hospital outpatient treatment. BMf 1990; 300: 1431-7.

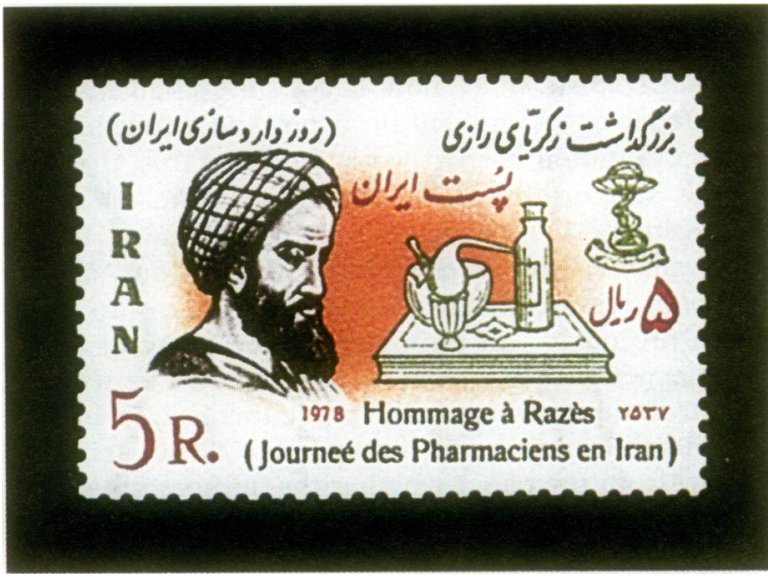

The golden age of Arab medicine produced great doctors, one of whom was Rhazes (born al-Razi) (AD 850-932). He was the author of about 237 books or treatises, of which 36 remain, dealing with science and medicine. He did not become a doctor until the age of 30 . He was interested in music, physics, philosophy, and alchemy. His capacity for observation and bold treatment earned him the title of 'the experimenter', though he followed the principles of Galen and Hippocrates. He became head of the great hospital at Baghdad and a physician of the Caliphs. As a proponent of a small therapeutic arsenal instead of the plethoric pharmacological fare of Arabian chemistry, he was quoted for over 500 years. His Liber Continens was an encyclopaedic collection of medical practice and treatment. The Liber Medicinalis ad Almansorem combined the corpus of the Greek, Syrian, and Arabian learning. He differentiated rheumatism from 'gout'. He also described spina-bifida. He noted that pain in the back would occur in smallpox but not in measles.

He is honoured on stamps of the Syrian Arabic republic and Iran. 